\title{
Asociación entre altitud geográfica e incidencia de rabia silvestre en ganado bovino en la región de Apurímac (2004-2015)
}

\author{
Association between geographical altitude and incidence of wild rabies in \\ cattle in the Apurimac region (2004-2015)
}

\author{
Martín Equicio Pineda Serruto ${ }^{1,4}$, Elisa María Vidal Cárdenas² ${ }^{2}$ Eric Humberto \\ Callapiña Enríquez ${ }^{3}$, Aldo Alim Valderrama Pomé ${ }^{1}$
}

\section{Resumen}

\begin{abstract}
La ganadería bovina latinoamericana se encuentra afectada por el virus de la rabia silvestre cuyo vector es el Desmodus rotundus. En 2014, Apurímac, Perú, registró el mayor número de casos $(\mathrm{n}=75)$. El objetivo del estudio fue determinar la asociación entre el tiempo calendario y los brotes de rabia silvestre bovina a distintas altitudes geográficas del valle de Apurímac, entre los años 2004 y 2015. Se analizaron datos del SIGSASENASA (Sistema Integrado de Gestión en Salud Animal del Servicio Nacional de Sanidad Agraria) sobre altitud geográfica $(\mathrm{m})$ y fecha de brotes sospechosos de rabia silvestre confirmada por inmunofluorescencia directa. Los datos fueron analizados mediante la prueba de Chi cuadrado, t-Student, correlación rho de Spearman y modelos lineales generalizados (GLM) de Poisson. Se analizaron 525 brotes sospechosos (rango de altitud: 999-4586 m), hallando diferencias para el número de brotes según altitudes: alta, media y baja $(\mathrm{p}<0.05)$. No se halló correlación entre altitud de brote y tiempo calendario ni asociación entre altitud geográfica del brote de rabia silvestre con tiempo calendario
\end{abstract}

\footnotetext{
${ }^{1}$ Facultad de Medicina Veterinaria y Zootecnia, Universidad Nacional Micaela Bastidas de Apurímac, Abancay, Apurímac, Perú

${ }^{2}$ Facultad de Salud Pública y Administración, Universidad Peruana Cayetano Heredia, Lima, Perú

${ }^{3}$ Área de Sanidad Animal, Servicio Nacional de Sanidad Agraria, Apurímac, Perú

${ }^{4}$ E-mail: mpineda@unamba.edu.pe; equicio29@hotmail.com
}

Recibido: 14 de diciembre de 2020

Aceptado para publicación: 10 de junio de 2021

Publicado: 24 de agosto de 2021

CLos autores. Este artículo es publicado por la Rev Inv Vet Perú de la Facultad de Medicina Veterinaria, Universidad Nacional Mayor de San Marcos. Este es un artículo de acceso abierto, distribuido bajo los términos de la licencia Creative Commons Atribución 4.0 Internacional (CC BY 4.0) [https:// creativecommons.org/licenses/by/4.0/deed.es] que permite el uso, distribución y reproducción en cualquier medio, siempre que la obra original sea debidamente citada de su fuente original 
ajustado por estacionalidad y tipo de crianza. Se concluye que hubo asociación entre el número de casos de rabia silvestre y la altitud geográfica $(\mathrm{p}<0.05)$, sin asociación al tiempo calendario; asimismo, se observó una asociación negativa con la temperatura mínima promedio $(\mathrm{p}<0.01)$.

Palabras clave: tiempo calendario, rabia bovina, Desmodus rotundus, quirópteros, altitud, Andes

\section{Abstract}

Latin American cattle farming is affected by the wild rabies virus whose vector is Desmodus rotundus. In 2014, Apurímac, Peru registered the highest number of cases $(\mathrm{n}=75)$ The objective of the study was to determine the association between calendar time and wild bovine rabies outbreaks at different geographical altitudes in the Apurímac valley, between the years 2004 and 2015. Data from the SIGSA-SENASA (Integrated Animal Health Management System of the National Agrarian Health Service) on geographical altitude (m) and date of suspected outbreaks of wild rabies confirmed by direct immunofluorescence were analysed. The data were analysed using the Chi square test, t-Student, Spearman's rho correlation and Poisson's generalized linear models (GLM). In total, 525 suspected outbreaks were analysed (altitude range: 999-4586 m), finding differences for the number of outbreaks according to altitudes: high, medium and low $(p<0.05)$. No correlation was found between outbreak altitude and calendar time or association between geographical altitude of the wild rabies outbreak with calendar time adjusted by seasonality and rearing system. It is concluded that there was an association between the number of cases of wild rabies and geographic altitude $(p<0.05)$, without association with calendar time. Likewise, a negative association was observed with the average minimum temperature $(\mathrm{p}<0.01)$.

Key words: calendar time, bovine rabies, Desmodus rotundus, chiropters, altitude, Andes

\section{INTRODUCCIÓN}

La geografía accidentada de los valles interandinos de Apurímac se caracteriza por cambios bruscos de altitud con pendientes pronunciadas a distancias cortas con muchas cuevas que facilitan la formación de nuevas colonias de murciélagos hematófagos. De esta manera, se facilita la aparición de zonas endémicas de rabia silvestre (García et al., 2004). Sin embargo, la expansión geográfica de Desmodus rotundus tiene como limitante la temperatura ambiental en determinados periodos del año (Lee, et al., 2012), donde además interviene la estacionalidad (George et al., 2011), y la altitud (m) (Cisneros et al.,
2014). Por otro lado, el tipo de crianza de ganado mejorado Holstein y Brown Swiss en los valles interandinos de Apurímac es predominantemente extensiva (INEI, 2012), mientras que el ganado criollo es de tipo trashumante (Herz, 2019), todo lo cual representa un hábitat propicio para la presentación de brotes de rabia silvestre (Maestri y Patterson, 2016).

En el Perú, la rabia silvestre bovina es una enfermedad endémica, habiéndose reportado la mayoría de los casos en el año 2014 $(n=242)$, especialmente en la región de Apurímac (75/242) (Vargas, 2015). En el continente americano, desde México hasta Argentina, la población del quiróptero Desmodus 
rotundus, vector del Lyssavirus causante de la rabia en ganado y humanos, se encuentra en aumento desde hace más de una década debido principalmente a su comportamiento migratorio (Blackwood et al., 2013; Torres et al., 2014; Escobar et al., 2015). Este fenómeno también se ha observado en los valles interandinos de Apurímac, localizándose el vector a distintas altitudes geográficas (García et al., 2004), posiblemente debido al aumento de la temperatura ambiental, como respuesta al fenómeno del calentamiento global (Lee et al., 2012). El Servicio Nacional de Meteorología e Hidrología (SENAMHI) y el Programa de Adaptación al Cambio Climático (PACC) del Gobierno Regional de Apurímac han reportado que las temperaturas promedio del departamento están cambiando a lo largo del tiempo (SENAMHI, 2012).

En Apurímac se ha tenido un incremento de brotes sospechosos de rabia silvestre entre 2003 y 2014 (Benavides et al., 2016), habiéndose observado que varios ganaderos del departamento han abandonado la actividad pecuaria por temor a contraer la rabia (Rubi Niño de Guzmán Montero, Apurímac, Comunicación personal). Esta situación está causando importantes pérdidas económicas y poniendo en riesgo la salud de las familias rurales (Navarro et al., 2007). Como respuesta al aumento de los brotes de rabia silvestre en el departamento (Benavides et al., 2016), el Servicio Nacional de Sanidad Agraria SENASA, solicitó al Gobierno Regional de Apurímac la ejecución de un Programa de Control de Rabia Silvestre, el cual se inició en 2014 y finalizó en setiembre de 2016.

En un estudio de modelamiento ecológico al este de los Andes del Perú para escenarios futuros (años 2030, 2050 y 2080), se estimó que por efecto del calentamiento global, la población de $D$. rotundus aumentará anualmente, abarcando nuevas zonas geográficas e incrementando los brotes de rabia silvestre en la ganadería de la zona (Lee et al., 2012). Sin embargo, el estudio se basó únicamente en datos meteorológicos de tem- peratura ambiental, sin considerar la altitud a la cual ocurrieron los brotes de rabia silvestre y la influencia en su dispersión geográfica (Blackwood et al., 2013). De esta manera, el presente estudio analizó la posible asociación entre la presencia de brotes de rabia y las altitudes geográficas considerando la temperatura ambiental a lo largo de 12 años (2004 al 2015) en la región de Apurímac, a fin de promover acciones efectivas de control (i.e. vacunación) en zonas vulnerables y que se adopten medidas estratégicas de vigilancia epidemiológica focal y perifocal por parte de entidades involucradas en la salud pública, como es el caso del SENASA y el Ministerio de Salud (MINSA) del Perú.

\section{Materiales y MéTodos}

\section{Diseño del Estudio}

Se analizaron datos secundarios facilitados por el Sistema Integrado de Gestión en Salud Animal del Servicio Nacional de Sanidad Agraria (SIGSA-SENASA). La base de datos contenía información relacionada al propietario, el predio, el tipo de ganado, la fecha de notificación del brote, la prueba de laboratorio realizada para el diagnóstico de rabia, y su resultado. Se realizó un estudio ecológico analítico del ámbito de los valles interandinos de Apurímac respecto a la presencia de rabia silvestre en el ganado durante el periodo 2004-2015. Los valles interandinos de Apurímac se caracterizan por ubicarse entre montañas con pendientes pronunciadas de variada altitud a distancias relativamente cortas y con una topografía accidentada con cuencas y microcuencas hidrográficas. El clima corresponde al de los valles interandinos de la cordillera oriental que varía por la altitud, siendo cálido y húmedo al pie de los cañones, templado y seco en las alturas medias, y frío con extrema sequedad en las cumbres de las montañas, con altitudes que se hallan entre 900 a $5000 \mathrm{~m}$. Respecto al contexto socio-demográfico, la rabia silvestre en Apurímac afecta a la ganadería de 
la zona principalmente bovina, afectando la sostenibilidad económica de los ganaderos (García et al., 2004).

\section{Población y Muestra}

La población de estudio estuvo constituida por ganado vacuno (Bos taurus), sin distinción de raza, sexo y edad, expuestos de manera natural al virus de la rabia silvestre. La muestra para el periodo 2004-2015 corresponde a 484 casos de rabia silvestre con resultados positivos en laboratorio. Estos casos corresponden a 462 notificaciones de brotes con uno a tres casos por brote, presentándose un solo caso en el $91.1 \%$ de las notificaciones. Dada la información que se cuenta de rabia por vigilancia pasiva según ubicación geográfica y fecha de ocurrencia y no conocerse la magnitud real de la presentación de los brotes, se usó el término de brote sospechoso en lugar de caso. La población de ganado vacuno mejorado en Apurímac, según el IV Censo Nacional Agropecuario de 2012 fue de 35593 cabezas (INEI, 2012).

\section{Procedimientos}

Brotes probables de rabia silvestre (desenlace): El SIGSA-SENASA registró la notificación de los brotes probables de rabia silvestre bovina mediante vigilancia pasiva, así como los casos de rabia silvestre confirmados en el laboratorio.

Muestras biológicas: En todo animal sospechoso de rabia (cojera, pérdida del equilibrio, desorientación) se esperaba la progresión natural de la enfermedad por 3-4 días hasta la postración del animal para proceder al sacrificio y la toma de muestra (cerebro, cerebelo e hipotálamo), tal como ha sido reportado (OIE, 2004). Las muestras fueron colocadas en bolsas de polietileno estéril, retirando el máximo de aire posible al cerrarla, se colocaban en termos con gel refrigerante para mantener la cadena de frío $\left(2-8^{\circ} \mathrm{C}\right), \mathrm{y}$ eran transportadas a las sedes del SENASA de Andahuaylas o Abancay. Las muestras fueron codificadas e ingresadas en la base de datos del SIGSA, y remitidas por vía aérea al Laboratorio Oficial de Referencia Nacional en Sanidad Animal, Unidad del Centro de Diagnóstico de Sanidad Animal (UCDSA) del SENASA en Lima. Las muestras fueron analizadas por medio de la prueba de inmunofluorescencia directa (IFD), prueba estándar para el diagnóstico de rabia según la Organización Mundial de la Salud (OMS) y la Organización Mundial de Sanidad Animal (Loza, 2004). La IFD tiene una sensibilidad de $100 \%$ y una especificidad de $94.3 \%$ para el diagnóstico de rabia (Ribas Antúnez et al., 2005).

Altitudes geográficas (exposición principal): Las coordenadas de los brotes sospechosos fueron ubicadas con el equipo de Sistema de Posicionamiento Global (GPS, siglas en inglés), utilizando el equipo Geoexplorer 3 y haciendo uso del software Google Earth Pro v. 7.1.2.2041 (Google Inc). Esta variable fue analizada como numérica a escala continua para la regresión lineal de Poisson. El análisis estadístico se realizó utilizando el software Stata v.14.0 (StataCorp LP).

\section{Otras Co-variables}

- Tiempo calendario: La base de datos del SIGSA registró las fechas de ocurrencia de infecciones por rabia silvestre. Para el presente estudio, se utilizó la variable tiempo en años, como una variable numérica en escala ordinal.

- Temperatura $\left(T^{\circ}\right)$ ambiental mínima promedio anual: Se utilizó esta variable como evidencia del cambio climático. Se recolectó la información para el periodo 2004-2015 en ocho estaciones meteorológicas de Apurímac localizadas entre 2000 y 4000 msnm del departamento de Apurímac. La información fue proporcionada por el SENAMHI.

- Estacionalidad: Corresponde a los periodos climáticos anuales de lluvia (entre octubre y marzo: semanas 1 a 13 y 40 a 52) y de seca (entre abril a setiembre: semanas 14 a 39), lo cual permitió dicotomizar la variable en estacionalidad de lluvia y seca. 
- Tipo de crianza: Los principales sistemas de crianza son i) extensiva, alimentación al pastoreo, ii) semi-extensiva, el alimento es suministrado de manera estabulada o confinada y al pastoreo, y iii) trashumancia, los animales permanecen libres en el monte (parte alta y con abundante vegetación) por periodos largos de tiempo (tres meses aproximadamente) sin ser vistos por los propietarios. Esta información fue registrada por el SIGSA en los hatos que reportaron probables brotes de rabia.

\section{Recolección de Datos}

El personal responsable de la toma de datos en las notificaciones fue un equipo conformado por un médico veterinario y un especialista en sanidad agraria, quienes cuentan con conocimientos en vigilancia epidemiológica. La toma de datos se registró en el formato oficial de notificaciones del SENASA, el cual incluye datos de ubicación del predio (dirección, nombre del establo, sector, localidad, distrito, provincia, departamento y extensión en hectáreas, latitud, longitud, altitud); fecha de la notificación (toma de muestra para laboratorio); datos del solicitante o propietario (documento nacional de identidad, nombre completo, teléfono y firma); y datos del animal sospechoso (identificación, edad, estado de producción, sexo y raza). Los datos fueron introducidos al SIGSA por el especialista en sanidad agraria dentro de las 24 horas de realizada la notificación.

\section{Plan de Análisis}

La relación entre ocurrencia de brotes y altitud según el tiempo calendario fue analizada por la correlación $r$ de Pearson para una potencia superior al $80 \%$ en una correlación esperada de 0.1 con un $n=484$ (brotes notificados), $\alpha=0.05$. Para el análisis bivariado se utilizó la prueba de Chi cuadrado para variables categóricas y Kruskal-Wallis para las continuas. Para la asociación brotes con altitud geográfica se usó el modelo lineal generalizado (GLM) de la familia Poisson y link log, ajustado por variables confusoras de temperatura ambiental y estacionalidad, usando el software de análisis estadístico Stata v. 14.0 (StataCorp LP).

\section{Consideraciones Éticas}

La fuente de datos provino del SENASA, entidad pública sin fines de investigación, por lo que no fue necesaria la aprobación ética. El acceso a la base de datos del SIGSA-SENASA fue entregada por el Jefe del Área de Sanidad Animal de Apurímac. Los datos correspondieron a animales cuyos propietarios notificaron la enfermedad al SENASA. Solo se tuvo acceso al código del propietario, quedando preservada la confidencialidad de la información que brindaron en la notificación. La toma de la muestra biológica del animal siguió el protocolo establecido, evitando causarle dolor innecesario. Este protocolo se registró en el Sistema Descentralizado de Información y Seguimiento a la Investigación (SIDISI) - Dirección Universitaria de Investigación, Ciencia y Tecnología (DUICT), y fue evaluado por el Comité de Ética de la UPCH (CIE-UPCH) previamente a su ejecución (Código 66950). Durante la implementación del estudio se respetaron los principios éticos delineados en la Declaración de Helsinki, y se siguieron estrictamente las recomendaciones realizadas por el CIE-UPCH.

\section{Resultados}

\section{Características Zootécnicas y Año}

El estudio fue realizado en ganado vacuno que presentó rabia transmitida por el murciélago $D$. rotundus en los valles interandinos de la Región Apurímac, entre altitudes de 999 a $4586 \mathrm{~m}$, con un total de 484 casos o brotes probables confirmados positivos por IFD entre 2004 a 2015. En ese periodo, la $\mathrm{T}^{\circ}$ mínima promedio anual estuvo entre 4.5 y $11.8^{\circ} \mathrm{C}$ y el $54.8 \%$ de brotes se presentó en la época de lluvia. El 96.9\% del ganado afectado era manejado bajo el siste- 
Cuadro 1. Factores asociados con la incidencia de casos confirmados de rabia silvestre en ganado vacuno de los valles interandinos de Apurímac durante el periodo 2004 a 2015

\begin{tabular}{|c|c|c|c|c|c|c|c|c|c|}
\hline \multirow[t]{2}{*}{ Características } & \multicolumn{3}{|c|}{ Análisis bivariado } & \multicolumn{3}{|c|}{$\begin{array}{l}\text { Regresión múltiple, } \\
\text { Modelo parsimónico }\end{array}$} & \multicolumn{3}{|c|}{$\begin{array}{l}\text { Regresión múltiple, } \\
\text { Modelos ajustados por } \\
\text { el parsimónico* }\end{array}$} \\
\hline & Coef. & $\begin{array}{l}\text { IC } \\
95 \%\end{array}$ & $\mathrm{p}$ & Coef. & $\begin{array}{l}\text { IC } \\
95 \%\end{array}$ & $\mathrm{p}$ & Coef. & $\begin{array}{l}\text { IC } \\
95 \%\end{array}$ & $\mathrm{p}$ \\
\hline \multicolumn{10}{|l|}{ Sexo } \\
\hline Macho & Ref. & & & Ref. & & & & & \\
\hline Hembra & 1.54 & $\begin{array}{l}1.29- \\
1.84\end{array}$ & 0.001 & 1.41 & $\begin{array}{l}1.18- \\
1.68\end{array}$ & 0.001 & & & \\
\hline \multicolumn{10}{|l|}{ Edad (meses) } \\
\hline $1-10$ & Ref. & & & & & & & & \\
\hline $11-20$ & 1.08 & $\begin{array}{l}0.82- \\
1.43\end{array}$ & 0.593 & 0.99 & $\begin{array}{l}0.75- \\
1.31\end{array}$ & 0.967 & & & \\
\hline$>20$ & 2.43 & $\begin{array}{l}1.94- \\
3.05\end{array}$ & 0.001 & 2.70 & $\begin{array}{l}2.15- \\
3.40\end{array}$ & 0.001 & & & \\
\hline \multicolumn{10}{|l|}{ Estacionalidad } \\
\hline Seca & Ref. & & & & & & Ref. & & \\
\hline Lluvia & 1.12 & $\begin{array}{l}0.94- \\
1.33\end{array}$ & 0.222 & & & & 1.19 & $\begin{array}{l}1.00- \\
1.42\end{array}$ & 0.055 \\
\hline \multicolumn{10}{|l|}{ Año calendario } \\
\hline $2004-2006$ & Ref. & & & & & & & & \\
\hline $2007-2009$ & 1.23 & $\begin{array}{l}0.91- \\
1.65\end{array}$ & 0.178 & 1.55 & $\begin{array}{l}1.15- \\
2.09\end{array}$ & 0.004 & & & \\
\hline $2010-2012$ & 0.97 & $\begin{array}{l}0.73- \\
1.30\end{array}$ & 0.837 & 1.57 & $\begin{array}{l}1.17- \\
2.10\end{array}$ & 0.003 & & & \\
\hline $2012-2015$ & 0.83 & $\begin{array}{l}0.62- \\
1.11\end{array}$ & 0.204 & 1.53 & $\begin{array}{l}1.14- \\
2.05\end{array}$ & 0.004 & & & \\
\hline \multicolumn{10}{|c|}{ Altitud geográfica } \\
\hline $500-2300$ & Ref. & & & Ref. & & & & & \\
\hline $2301-3500$ & 4.04 & $\begin{array}{l}3.11- \\
5.24\end{array}$ & 0.001 & 4.63 & $\begin{array}{l}3.56- \\
6.03\end{array}$ & 0.001 & & & \\
\hline $3501-4000$ & 0.58 & $\begin{array}{l}0.33- \\
1.03\end{array}$ & 0.064 & 0.50 & $\begin{array}{l}0.28- \\
0.90\end{array}$ & 0.020 & & & \\
\hline $4001-4800$ & 0.61 & $\begin{array}{l}0.33- \\
1.12\end{array}$ & 0.113 & 0.81 & $\begin{array}{l}0.43- \\
1.50\end{array}$ & 0.498 & & & \\
\hline
\end{tabular}

* Ajustado por las variables altitud geográfica, edad, sexo y año calendario. 
Cuadro 2. Características zootécnicas y temporales de los casos confirmados de rabia silvestre en ganadería bovina de los valles interandinos de Apurímac

\begin{tabular}{|c|c|c|c|c|}
\hline \multirow{2}{*}{$\begin{array}{l}\text { Factores de } \\
\text { asociación }\end{array}$} & \multicolumn{3}{|c|}{ Altitud del brote de rabia silvestre } & \multirow[b]{2}{*}{ p-valor* } \\
\hline & $\begin{array}{c}\text { Alta } \\
(\mathrm{n}=175)\end{array}$ & $\begin{array}{c}\text { Media } \\
(\mathrm{n}=173)\end{array}$ & $\begin{array}{c}\text { Baja } \\
(\mathrm{n}=177)\end{array}$ & \\
\hline Sexo & & & & 0.628 \\
\hline Hembra & $101(34.0)$ & $101(34.0)$ & $95(32.0)$ & \\
\hline Macho & $74(32.4)$ & $72(31.6)$ & $82(36.0)$ & \\
\hline Edad (meses) $\dagger$ & $24(12-36)$ & $24(12-36)$ & $24(12-36)$ & 0.484 \\
\hline Tipo de crianza & & & & 0.387 \\
\hline Extensiva & $167(32.8)$ & $169(33.2)$ & $173(34.0)$ & \\
\hline Semi-extensiva & $5(45.4)$ & $4(36.4)$ & $2(18.2)$ & \\
\hline Trashumancia & $3(60.0)$ & $0(0.0)$ & $2(40.0)$ & \\
\hline Año calendario & & & & 0.004 \\
\hline 2005 & $9(42.8)$ & $6(28.6)$ & $6(28.6)$ & \\
\hline 2006 & $16(37.2)$ & $16(37.2)$ & $11(25.6)$ & \\
\hline 2007 & $12(34.3)$ & $14(40.0)$ & $9(25.7)$ & \\
\hline 2008 & $21(35.6)$ & $27(45.8)$ & $11(18.6)$ & \\
\hline 2009 & $5(12.5)$ & $18(45.0)$ & $17(42.5)$ & \\
\hline 2010 & $17(27.0)$ & $0(31.7)$ & $26(41.3)$ & \\
\hline 2011 & $18(34.0)$ & $14(26.4)$ & $21(39.6)$ & \\
\hline 2012 & $8(21.0)$ & $11(29.0)$ & $19(50.0)$ & \\
\hline 2013 & $24(44.4)$ & $7(13.0)$ & $23(42.6)$ & \\
\hline 2014 & $24(32.0)$ & $26(34.7)$ & $25(33.3)$ & \\
\hline 2015 & $21(47.7)$ & $14(31.8)$ & $9(20.5)$ & \\
\hline Estacionalidad (época) $\dagger \dagger$ & & & & 0.019 \\
\hline $\begin{array}{l}\text { Lluvia (octubre- } \\
\text { marzo) }\end{array}$ & $82(28.9)$ & $107(37.7)$ & $95(33.4)$ & \\
\hline $\begin{array}{l}\text { Seca (abril - } \\
\text { setiembre) }\end{array}$ & $93(38.6)$ & $66(27.4)$ & $82(34.0)$ & \\
\hline
\end{tabular}

* Chi cuadrado

+ Mediana (rango intercuartil)

†† Estacionalidad, cada estación dura 26 semanas o seis meses: Lluvia (semana 1-13 y 40-52) y Seca (14-39)

ma de crianza extensiva. La mediana de edad del ganado fue de 24 meses (rango intercuartil: 12 a 36 meses), y el 56.8\% fueron hembras. Asimismo, la mayor cantidad de brotes sospechosos notificados ocurrió en 2014, año en que se registró el $13.4 \%$ de los 484 brotes (Cuadro 2).
De otra parte, ninguna de las variables como tiempo calendario, estacionalidad y tipo de crianza mostraron diferencias respecto a la altitud de ocurrencia de los brotes de rabia silvestre $(\mathrm{p}>0.05)$, teniendo como variable continua el año calendario. De estos análisis por GLM, tampoco se hallaron asociaciones 


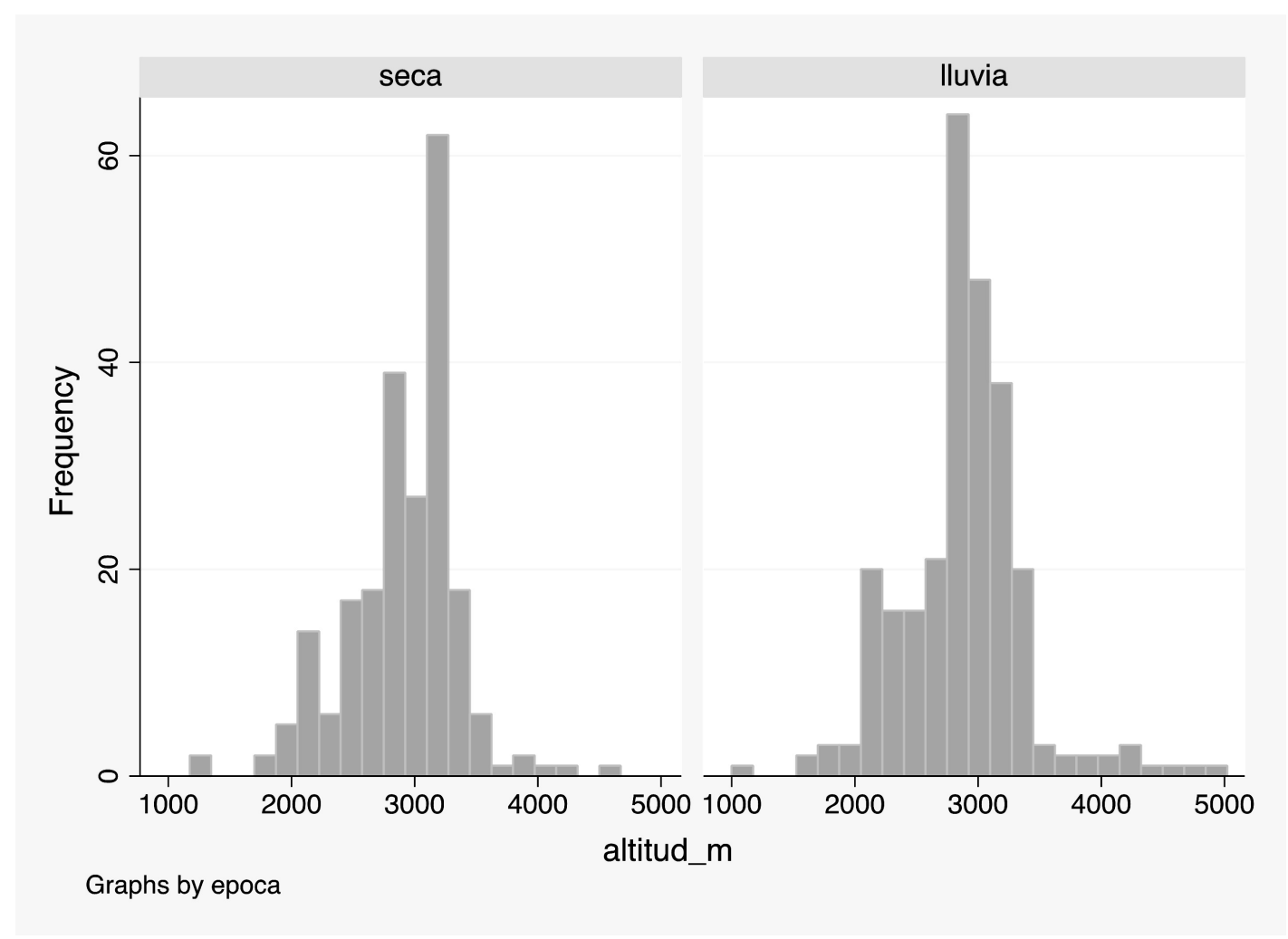

Figura 1. Frecuencia de casos de rabia silvestre confirmada por inmunofluorescencia directa (IFD) en ganado bovino de los valles interandinos de Apurímac por altitud geográfica (2004 - 2015) y estacionalidad

entre terciles de altitud geográfica de la ocurrencia de brotes de rabia silvestre y el tiempo calendario o la estacionalidad o el tipo de crianza del ganado $(\mathrm{p}>0.05)$ (Cuadro 1$)$.

\section{Altitud de Ocurrencia}

Las altitudes geográficas de la ocurrencia de brotes de rabia silvestre en terciles de alta, media y baja altitud varían en el tiempo calendario o por estacionalidad posiblemente por la topografía particular que caracteriza a los valles interandinos (Figura 1).
Durante la estación de lluvias se observó que el mayor número de brotes ocurrió en altitud media (tercil 2) (37.7\%), mientras que durante la estación seca se presentó en altitud alta (tercil 3) (38.6\%). Durante el periodo 2004-2015, el mayor porcentaje de brotes probables para el tercil de alta altitud ocurrió en 2015 (47.7\%), siendo superior al de las alturas media $(31.8 \%)$ y baja $(20.5 \%)$. Las variables tiempo (en años) y estacionalidad (épocas de lluvia y seca) según los terciles de altitud geográfica (alta, media o baja) mostraron variación estadística significativa 


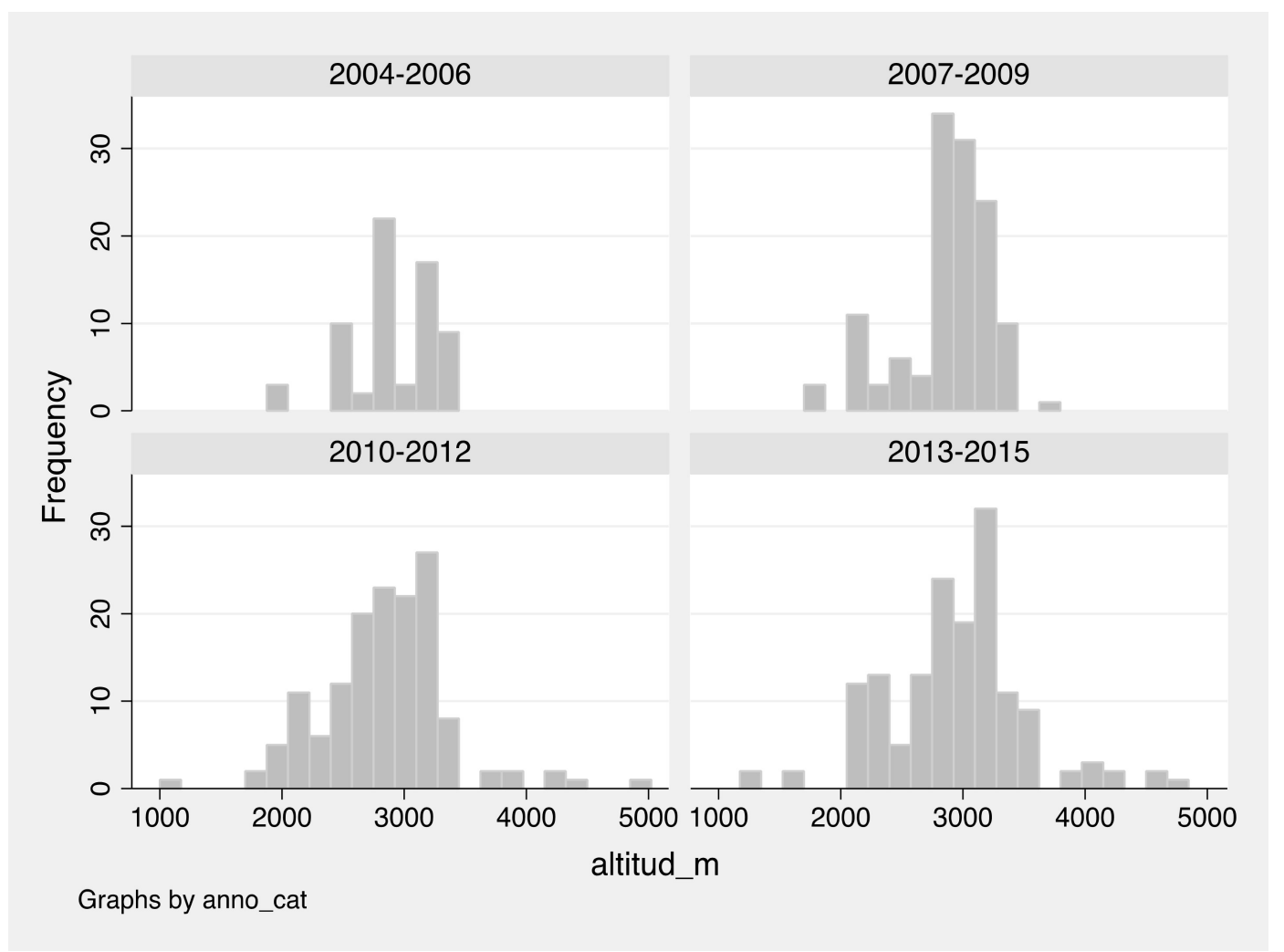

Figura 2. Frecuencia de casos de rabia silvestre confirmada por inmunofluorescencia directa (IFD) en ganado bovino de los valles interandinos de Apurímac por altitud geográfica

$(\mathrm{p}<0.05$; Figura 2$)$; sin embargo, no se observó una variación estadística entre las variables sexo, edad y tipo de crianza del ganado respecto al número de brotes por terciles de altitud geográfica $(\mathrm{p}>0.05)$ (Cuadro 2).

\section{Discusión}

El presente estudio describe la distribución de rabia silvestre en la región de Apurímac en el periodo 2004-2015, tanto en altitudes altas como medias y bajas. Se encontró que la tasa de incidencia de rabia en altitudes entre 2301 y $3500 \mathrm{~m}$ es cuatro veces la tasa de incidencia que se presenta en altitudes entre 500 y $2300 \mathrm{~m}(\mathrm{p}=0.001)$. Se estipula que el cambio en la distribución de la rabia silvestre está asociado al cambio climático (Lee et al., 2012), en donde los patrones de temperatura en Apurímac sobre los $3500 \mathrm{msnm}$ han cambiado, incrementándose la temperatura media mínima en época de invierno (SENAMHI, 2012). Este hecho también es afectado por la estacionalidad característica de una época seca con condiciones de temperatura ambiental favorable para el vector (Rocha y Dias, 2020); así, la actividad hematófaga del vector ocurre cuando el clima está despejado, es decir, será mayor en la estación seca y por el largo periodo de incubación del Lyssavirus muchos casos serán notificados recién en época de lluvia. Contrariamente el menor número de mordeduras en el ganado por $D$. rotundus en la estación de lluvia que se presentan en los periodos cortos de clima despejado, 
devendrá en notificaciones para la estación seca (da Rocha et al., 2018), pero, ocurrirán en menor proporción que en época de lluvia.

Asimismo, los refugios son parte del hábitat del murciélago y son propios de la geografía de los valles interandinos (Barros et al., 2020). La presencia de refugios permite al vector establecer nuevas colonias, por lo cual el número de refugios presentes a altitudes bajas, medias y altas podría explicar parte de la magnitud de la asociación entre brotes de rabia por altitud geográfica.

La zona climática con mayor riqueza biológica se halla alrededor de los $2500 \mathrm{msnm}$ (Escobar et al., 2015), lo que favorecería al vector, posibilitando un mayor número de brotes de rabia silvestre en el ganado a esta altitud. La incidencia de casos de rabia fue menor en el periodo 2004-2006, aumentando con los años, posiblemente debido a la expansión del vector, así como al incremento de la crianza de ganado lechero mejorado. La variable confusora, condición meteorológica de temperatura mínima, podría explicar la magnitud de la frecuencia de rabia a través de los años del estudio. Además, la temperatura ambiental es una variable que está directamente ligada al piso altitudinal y la latitud geográfica (INDECI, 2012). Dado que el índice de mordedura del vector D. rotundus por animal y por hato se relaciona directamente con brotes confirmados de rabia silvestre en el ganado, donde a mayor índice de mordedura mayor número de casos positivos, se puede considerar que a pesar de no tener información sobre la presencia de refugios a distintas altitudes (baja, media, alta) ni del índice de mordedura, estos hayan afectado los resultados (Vargas, 2015).

La distribución espacial de rabia silvestre se ha ido expandiendo a nuevas zonas geográficas (Sistema Nacional de Vigilancia Epidemiológica, 2014). Sin embargo, la presencia de brotes probables de rabia silvestre a diferentes niveles de altitud (baja, media, alta) no estuvo asociada con la época del año (lluvia, seca) en los valles de la región de
Apurímac, de allí que la ocupación de nuevas zonas geográficas por $D$. rotundus a través de los años se da independientemente de la época del año (lluvia, seca).

Las altitudes en que ocurren los brotes de rabia silvestre no estuvieron asociadas estadísticamente al tiempo calendario ( $p>0.05$ ), pero se observó asociación negativa con la temperatura mínima promedio $(p=0.001)$. El SENASA y MINSA deben establecer medidas de vigilancia epidemiológica preventiva. Es necesario estudiar la rabia silvestre y sus implicancias económicas. Es importante que los estudios futuros se orienten en brindar información que ayude al control del vector, de manera de replantear las estrategias que viene implementando el SENASA para la reducción efectiva de los casos de rabia silvestre.

\section{Conclusiones}

- Se encontró asociación entre número de casos de rabia silvestre y altitud geográfica, siendo la tasa de incidencia en altitud de 2301-3500 m cuatro veces la tasa de incidencia que se presenta en 500$2300 \mathrm{~m}$.

- Las frecuencias de casos en los periodos 2007-2009, 2010-2012 y 2013-2015 fueron 50\% superiores al periodo 20042006.

- La estacionalidad no influyó en la presentación de casos de rabia silvestre.

- Se encontró asociación negativa con la temperatura mínima promedio.

\section{Literatura Citada}

\section{Barros $J$ de $S$, Bernard E, Ferreira}

RL. 2020. Ecological preferences of neotropical cave bats in roost site selection and their implications for conservation. Basic Appl Ecol 45: 31-41. doi: 10.1016/j.baae.2020.03.007 
2. Benavides JA, Valderrama W, Streicker DG. 2016. Spatial expansions and travelling waves of rabies in vampire bats. P R Soc B 283: 20160328. doi: 10.1098/rspb.2016.0328

3. Blackwood JC, Streicker DG, Altizer S, Rohani P. 2013. Resolving the roles of immunity, pathogenesis, and immigration for rabies persistence in vampire bats. P Natl Acad Sci USA 110: 20837-20842. doi: 10.1073/pnas.1308817110

4. Cisneros LM, Burgio KR, Dreiss LM, Klingbeil BT, Patterson BD, Presley SJ, Willig MR. 2014. Multiple dimensions of bat biodiversity along an extensive tropical elevational gradient. J Anim Ecol 83: 1124-1136. doi: 10.1111/ 1365-2656.12201

5. da Rocha PA, Ruiz-Esparza J, Ferrari $S F$. 2018. Differences in the structure of the bat community between a cloud forest refuge and a surrounding semi-arid Caatinga scrubland in the northeastern Brazil. J Arid Environ 151: 41-48. doi: 10.1016/j.jaridenv.2017.11.005

6. Herz CA. 2019. Procesos endógenos en la sostenibilidad de los sistemas de producción de camélidos en regiones altoandinas del sur de Perú: caso Cotaruse, Apurímac. Tesis Doctoral. Lima, Perú: Pontificia Universidad Católica del Perú. 248 p.

7. [OIE] Organización Mundial de Sanidad Animal. 2004. Manual de la OIE sobre animales terrestres. París, Francia. [Internet]. Disponible en: https:// www.oie.int/fileadmin/Home/esp/ Publications_\&_Documentation/docs/ pdf/2.2.05_Rabia.pdf

8. Escobar LE, Peterson AT, Pape M, Favi M, Yung V, Restif O, Qiao $\mathrm{H}$, et al. 2015. Ecological approaches in veterinary epidemiology: mapping the risk of bat-borne rabies using vegetation indices and night-time light satellite imagery. Vet Res 46: 92. doi: 10.1186/ s13567-015-0235-7
9. George DB, Webb CT, Farnsworth ML, O'Shea TJ, Bowen RA, Smith DL, Stanley TR, et al. 2011. Host and viral ecology determine bat rabies seasonality and maintenance. P Natl Acad Sci USA 108: 10208-10213. doi: 10.1073/ pnas. 1010875108

10. García F, Holgado W, Aguilar EG, Quispe MM. 2004. IFD para la detección del virus de la rabia en murciélagos del distrito de Huanipaca - Abancay. Situa 13: 5-9.

11. George DB, Webb CT, Farnsworth ML, O'Shea TJ, Bowen RA, Smith DL, et al. 2011. Host and viral ecology determine bat rabies seasonality and maintenance. Proc Ntl Acad Sci USA 108: 10208-10213. doi: 10.1073/ pnas. 1010875108

12. Herz CA. 2019. Procesos endógenos en la sostenibilidad de los sistemas de producción de camélidos en regiones altoandinas del sur de Perú: caso Cotaruse, Apurímac. Tesis Doctoral. Lima: Pontificia Universidad Católica del Perú. $248 \mathrm{p}$.

13. [INDECI] Instituto Nacional de Defensa Civil. 2012. La gestión del riesgo de desastres en el Perú. Documento país Perú 2012. Lima: INDECI. Plan de acción DIPECHO 2011-2012. 146 p.

14. [INEI] Instituto Nacional de Estadística e Informática. 2012. IV Censo Nacional Agropecuario. Resultados Definitivos. IV Censo Nacional Agropecuario. [Internet]. Disponible en: http:// proyectos.inei.gob.pe/web/DocumentosPublicos/ResultadosFinalesIVCENAGRO.pdf

15. Lee DN, Pape M, van Den Bussche $R A$. 2012. Present and potential future distribution of common Vampire bats in the Americas and the associated risk to cattle. Plos One 7: e42466. doi: 10.1371/ journal.pone. 0042466 
16. Maestri R, Patterson BD. 2016. Patterns of species richness and turnover for the South American rodent fauna. Plos One 11: e0151895. doi: 10.1371/ journal.pone. 0151895

17. Navarro AM, Sato A, Bustamante J. 2007. Situación actual y control de la rabia en el Perú. Rev Peru Med Exp Salud Publica 24: 46-50.

18. [OIE] Organización Mundial de Sanidad Animal. 2004. Manual de la OIE sobre animales terrestres. París: OIE. [Internet]. Disponible en: https:// www.oie.int/fileadmin/Home/esp/ Publications_\&_Documentation/docs/ pdf/2.2.05_Rabia.pdf

19. Ribas Antúnez MA, Acosta Renom G, Tejero Suárez Y, García Martínez D, Rodríguez Valdez C. 2005. Estudio comparativo entre 2 conjugados para el diagnóstico por inmuno-fluorescencia directa de la rabia en Cuba. Rev Cubana Med Trop 57: 120-124.
20. Rocha F, Dias RA. 2020. The common vampire bat Desmodus rotundus (Chiroptera: Phyllostomidae) and the transmission of the rabies virus to livestock: A contact network approach and recommendations for surveillance and control. Prev Vet Med 174: 104809. doi: 10.1016/j.prevetmed.2019.104809

21. [SENAMHI] Servicio Nacional de Meteorología e Hidrología. 2012. Caracterización climática de las regiones Apurímac y Cusco. Lima: SENAMHI. Informe final de investigación del estudio bi-regional disciplinario realizado. $113 \mathrm{p}$.

22. Torres C, Lema C, Dohmen FG, Beltran F, Novaro L, Russo S, Freire $M C$, et al. 2014. Phylodynamics of vampire bat-transmitted rabies in Argentina. Mol Ecol 23: 2340-2352. doi: $10.1111 /$ mec. 12728

23. Vargas E. 2015. Situación de la rabia en el Perú. Boletín Epidemiológico 24(7): 146-150. 